\title{
A High Position Resolution X-Ray Detector: An "Edge on" Illuminated Capillary Plate Combined With a Gas Amplification Structure
}

\author{
C. Iacobaeus, T. Francke, B. Lund-Jensen, J. Ostling, P. Pavlopoulos, V. Peskov, and F. Tokanai
}

\begin{abstract}
We have developed and successfully tested a prototype of a new high position resolution hybrid $X$-ray detector. It contains a thin-wall lead glass capillary plate converter of $X$-rays combined with a microgap parallel-plate avalanche chamber operating in various gas mixtures at 1 atm.

The operation of these converters was studied in a wide range of $\mathrm{X}$-ray energies (from 6 to $60 \mathrm{keV}$ ) at incident angles varying from $0-90^{\circ}$. The detection efficiency, depending on the geometry, photon's energy, incident angle and the mode of operation was between 5-30\% in single step mode and up to $50 \%$ in a multi-layered combination. Depending on the capillary's geometry, the position resolution achieved was between $50-250 \mu \mathrm{m}$ in digital form and was practically independent of the photon's energy or gas mixture. The usual lead glass capillary plates operated without noticeable charging up effects at counting rates of $50 \mathrm{~Hz} / \mathrm{mm}^{2}$ and hydrogen treated capillaries-up to $10^{5} \mathrm{~Hz} / \mathrm{mm}^{2}$. The developed detector has several important potential advantages over the exciting X-ray detectors and may open new possibilities for medical imaging, for example in mammography, portal imaging, radiography (including security devices), as well as many other applications.
\end{abstract}

Index Terms-Capillary plates, gaseous detectors, mammography, X-ray imaging.

\section{INTRODUCTION}

$\mathbf{I}$ $\mathrm{N}$ the last few decades a revolution took place in the X-ray imaging techniques: traditional film-based detectors are now being replaced by electron imaging devices. Currently there are two main directions in these developments: "static " two-dimensional (2D) detector systems (or a 2D matrix of microdetectors) [1] and one-dimensional (1D) scanning systems (or an 1D matrix of micro detectors) — see for example [2] and references therein. Today clinics have various commercial 2D sys-

Manuscript received October 21, 2004; revised December 12, 2005.

C. Iacobaeus is with the Department of the Medical Radiation Physics Karolinska Institute, Stockholm S-17176, Sweden (e-mail: Christian@radfys.ki.se).

T. Francke is with the XCounter AB, Danderyd S-18233, Sweden (e-mail: tom.francke@xcounter.se).

B. Lund-Jensen is with the Particle Physics Department of the Royal Institute of Technology, Stockholm S-10691, Sweden (e-mail: lund@ particle.kth.se).

J. Ostling is with the Medical Radiation Physics Department at Stockholm University, Stockholm, S-10691, Sweden (e-mail: ostling@particle.kth.se).

P. Pavlopoulos is with the Research Division at the Leonardo de Vinci University, Cedex 92916, Paris, France, and also with CERN, Geneva CH-1211, Switzerland (e-mail: Noulis.Pavlopoulos@devinci.fr).

V. Peskov is with the Research Division at the Leonardo de Vinci University, Cedex 92916, Paris, France (e-mail: vladimir.peskov@ cern.ch).

F. Tokanai is with the Physics Department of the Yamagata University, Yamagata 990-8560, Japan (e-mail: tokanai@sci.kj.yamagata-u.ac.jp).

Digital Object Identifier 10.1109/TNS.2006.872635 tems, based for example on scintillators readout by a CCD or CsI (Tl) deposited upon a large area arrays of photodiodes and readout switches fabricated on amorphous Si [2].

The main advantages of the 2D detectors are: a compact planar geometry and a short time required for the image taking. The disadvantages however are the additional noise due to the sensitivity to the scattered radiation and the high cost. Note that most of the $2 \mathrm{D}$ commercial devices are operating in a charge integration mode when the charge produced in the sensitive element by the radiation is accumulated in pixels for some time before being readout.

The main tendency in current laboratory developments is to implement a photon counting technique [3] which has potential for improvement of the dynamic range in the contrast of the observed X-ray images [4]. Photon counting however puts large demands on the detector's technology. The photons in the high X-ray flux should be time-resolved and be detected and counted one by one with high efficiency and high position resolutions. This should be done for a large number of pixels simultaneously and a high rate. It is clear that the cost of these advanced devices will remain very high.

In scanning systems the X-ray source is collimated to a narrow beam which is scanned across the human body or any other object in synchrony with a ID detector which measures the transmission radiation. Scanning systems allow a very efficient rejection of the scattered radiation, improving contrast and the signal to noise ratio. The other important feature of the scanning systems is that they have much simpler electronics and as a result the possibility arises of simultaneous photon counting and measuring their energy. The cost of the scanning system is usually much lower than for the $2 \mathrm{D}$ imagers.

Nowadays, two types of scanning systems are under development and clinical testing: base on solid -state detectors and based on high pressure gaseous detectors.

A linear matrix of solid-state detectors (for example $\mathrm{Si}$ or GaAs [5]) is very attractive for mammographic applications, however, in the case of the large-scale detectors oriented on a full body scan, this approach remains very expensive.

In contrast, gaseous detectors are relatively cheap and are almost noise free if operated at high avalanche gains [6]. This allows one to reduce the dose delivered to the patient during the examination [7]. However, the stopping power of gases is very low and this restricts the application of the gaseous detectors.

The only way to increase the efficiency the gaseous detector is to make it operate at an elevated pressure, this however, makes 
their design rather complicated and bulky and finally considerably increases their cost. In spite of these difficulties there are continuous efforts from various groups to develop high pressure gaseous detectors and implement them for X-ray scanning systems. A good example could be the multiwire proportional wire chambers (MWPC's) operating at pressures of only a few atm and which are currently used in some clinics [8]. Such detectors have modest position resolutions $(0.3-0.6 \mathrm{~mm})$, but operate in photon counting modes and are able to obtain high quality images at the reduced doses delivered to the patient. To manufacture a position-sensitive detector operating in avalanche mode at pressures high than a few atm is a real technical challenge due to the very high working voltages and other problems such as bulky feedthroughs and gas purity [9]. For this reason most of the existing detectors filled with gases at pressures of $10 \mathrm{~atm}$ and more operate in ionization chamber modes only. But even at these pressures the position resolution is limited (typically by 200-300 $\mu \mathrm{m}$ ) due to the electron diffusion, X-ray fluorescent photons and several other effects (see for example [10] and references therein).

The invention of the micropattern gaseous detectors (see [11] and references therein) gave a new momentum for the development in the scanning systems equipped with 1D gaseous detectors operating in avalanche modes. Micropattern gaseous detectors have high-segmented rigid electrodes and they operate at much lower low voltages than the MWPC which is an encouraging argument for their use at elevated pressures. Inspired by this idea several groups developed laboratory prototypes of $\mathrm{X}$-ray scanners which allow one to achieve position resolutions of a few times higher than the wire chambers [6], [12], [13]. However, during these developments it was discovered that the maximum achievable gain of any micropattern detector drops rapidly with the pressure [11], [14], so that at $\mathrm{p}>10 \mathrm{~atm}$ it was very difficult to achieve a noise free operation in photon counting mode.

Recently we have developed innovative designs of micropattern gaseous detectors in which X-rays were converted to the primary electrons either in an CsI layer or in a capillary plate [15]. These devices allowed one to achieve a position resolution of 30-50 $\mu \mathrm{m}$ with a planar electrode coated by an CsI layer [15] and about $300 \mu \mathrm{m}$ with the capillary plates [16]. The main breakthrough was that this extraordinary position resolution was achieved not at a high pressure as in the case of the earlier quoted works ([6], [8], [12], [13]), but at 1 atm. This tremendously simplifies the gas chamber's design, allowing one to use cheap gases (such as Ar-based mixtures) and even allowing one to flush the chamber with the gas instead of using expensive sealed chambers. The other important feature was that $30 \mu \mathrm{m}$ position resolutions with the CsI converters were obtained in simple counting (digital) modes without any application of the "centre of gravity" method or any other analog-interpolation method. This significantly simplifies the electronics and allows one to obtain images at very high counting rates: up to $10^{5} \mathrm{~Hz}$ per strip. It was an almost ideal device for the scanner oriented on soft X-ray imaging such as mammography. The efficiency, however, for hard X-rays (for example for $60 \mathrm{keV}$ ) was low [16].

The aim of this work is to develop a prototype of a new advanced $1 \mathrm{D}$ gaseous detector for scanner systems which com- bines high position resolutions with a high efficiency for hard $\mathrm{X}$-rays and can operate in gas mixtures at $\mathrm{p}=1 \mathrm{~atm}$.

After several preliminary tests and studies we finally chose as our main approach a combination of a capillary plate with a microgap gas amplification structure. However, in contrast to our previous works in this study we used thin-wall lead glass capillary plates (CP's) in an edge-on illuminated mode which allowed a high efficiency to be achieved. This paper describes our first results obtained in this very promising direction.

\section{Why Thin- Wall CPs ARE EFFICIENT X-RaY CONVERTERS?}

Works of Del Guerra, Lim, Ortendahl, Perez-Mendez et al., [17] and Sakurai, Tamura, Gunj and Norma [18] triggered the use of CP's as planar amplification structures in gaseous detectors. In most cases the CP's used were $0.8 \mathrm{~mm}$ thick, had diameter of active area of $20 \mathrm{~mm}$, the hole's radius of $50 \mu \mathrm{m}$, the wall thickness of $20-35 \mu \mathrm{m}$, and an open area ratio of $57 \%$. As was shown in [19] this geometry allows one to achieve the highest gas gains possible.

Recent attempts to use CP's as X-ray converters combined with various gas amplification structures are described in [15], [16]. In these tests the CP's used had the same geometry as described above and were illuminated by X-rays from the "top"-almost perpendicular to their flat electrode's surfaces. However, the geometry of the $\mathrm{CP}$ which is optimal for the avalanche multiplication is not necessary optimal for the X-ray conversion and detection. Indeed, when X-rays interact with the CP's material, a fast electron (in lead glass a photoelectric effect dominates) and a swarm of delta electrons will be created inside the material. Among these electrons only a small part of them, which are very close to the wall's surface (on a distance less than some characteristical distance d), could escape into the gas and create a track of primary electrons there (see Fig. 1(a)). By the influence of the applied electric field the primary electrons from this track cold drift in the gas inside the holes and be extracted from the holes to the external amplification structure.

The efficiency of this converter for the X-ray with the energy of $\mathrm{E}_{\mathrm{v}}$ could be defined as

$$
\eta=B_{\mathrm{tr}} / B_{\mathrm{ph}}
$$

where $B_{t r}$ is the number of tracks created in the gas and $B_{p h}$ is the number of incident photons per unit of area $\left(\mathrm{cm}^{2}\right)$.

One can make the following simplified qualitative estimation for the $\mathrm{B}_{\mathrm{tr}}$ for the CP's illuminated from the top

$$
B_{\mathrm{tr}} \sim B_{\mathrm{ph}} 2 \pi \mathrm{rdH}\left(1-e^{-K L}\right)
$$

where $\mathrm{L}$ is the CP's thickness, $\mathrm{K}$ is a linear absorption coefficient for the X-rays with the energy $\mathrm{E}_{\mathrm{v}}, \mathrm{r}$ is the radius of the CP's holes, $\mathrm{H}$ is the number of holes per unit of area, $\mathrm{d}$ is the thickness of the CP's surface layer from which the photoelectrons or delta electrons can escape into the gas $(\mathrm{d} \ll r)$. Because for the CP's the open area ratio is usually around $50 \%\left(\mathrm{H} \pi \mathrm{r}^{2} \sim 1 / 2\right)$, one can make the following estimations: the distance between the hole's center is $\sim 2.5 \mathrm{r}$ and the minimum thickness of the wall between the capillaries is $\mathrm{D} \approx 0.5 \mathrm{r}$. One can further assume that the optimal geometry for reaching the highest efficiency will be 


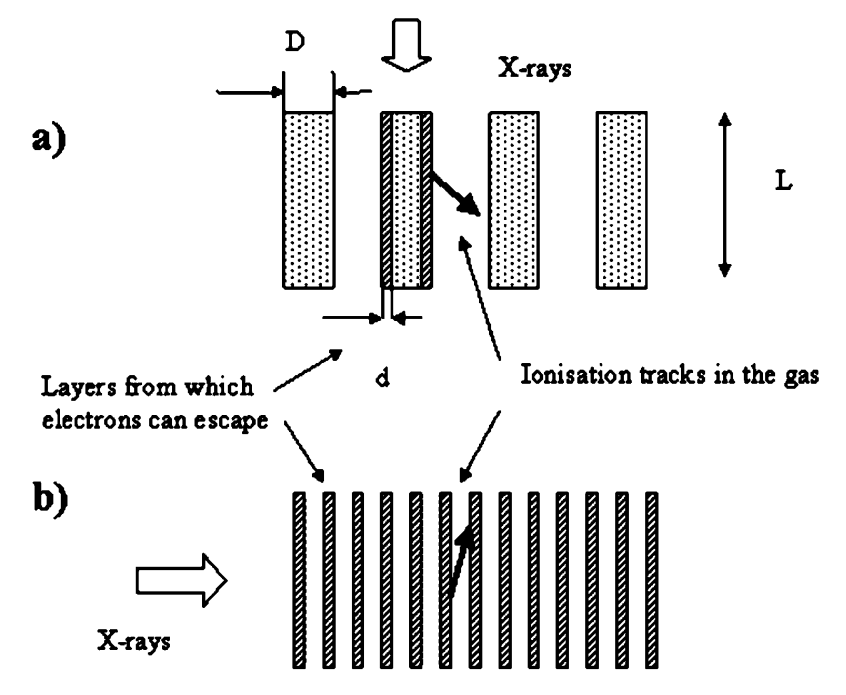

$2 \mathrm{R}$

Fig. 1. Schematic drawing (cross-section) of a thick-wall CP illuminated by $\mathrm{X}$-rays from the top (a) and a thin- wall edge-on illuminated CP (b).

when $\mathrm{D} \approx 2 \mathrm{~d}$. By putting these approximate values into the formulas (1-2) one will obtain the following (very simplified) estimation for the efficiency of the CP illuminated from the top:

$$
\eta_{\text {top }} \sim 0.25\left(1-e^{K L}\right)
$$

As one can see from these qualitative considerations, the efficiency of the CP's illuminated from the top has a clear limit: $\eta<25 \%$. This is due to the fact that $\sim 50 \%$ of the incident photons will pass the $\mathrm{CP}$ via its holes without any interactions. The other 50\% will hit the CP's material but only a small fraction of them, created in the layer with the thickness of $d$ around the capillary hole, will be able to create a track in the gas which could be then detected.

In contrast one can expect that the efficiency of the thin-wall edge-on illuminated CP's with $\mathrm{d} \approx 0.25 \mathrm{r}$ could be considerably higher due to the much larger efficient thickness for the $\mathrm{X}$-ray absorption as compared to the top illuminated CP (2R " d) - see Fig. 1(b). Indeed, the efficiency of the edge-on illuminated CP (for the pencil-beam collimated X-ray aligned along the diameter of the $\mathrm{CP}$ ) could be estimated as follows:

$$
\eta_{\text {edge }} \sim 1-e^{-K D n 2 R}=1-e^{-4 K d n R}
$$

where $\mathrm{n}$ is the number of holes per unit of length of the $\mathrm{CP}$ and $\mathrm{R}$ is the radius of the CP. Since $n^{2} \sim \mathrm{H}$ one can rewrite the (4) as follows:

$$
\eta_{\text {edge }} \sim 1-e^{-K R \sqrt{ }(0.5 / \pi)}
$$

Because of $\mathrm{R} \gg \mathrm{L}$, it follows that $\eta_{\text {edge }}>\eta_{\text {top }}$.

Practical conclusions which one can make from these simplified considerations are the following.

1) In contrasts to the CP's used as gas amplification structures (hole diameters of $100 \mu \mathrm{m}$ ) the $\mathrm{CP}$ in the X-ray conversion should have much smaller diameters of the holes $(\mathrm{r} \approx 4 \mathrm{~d}<100 \mu \mathrm{m})$

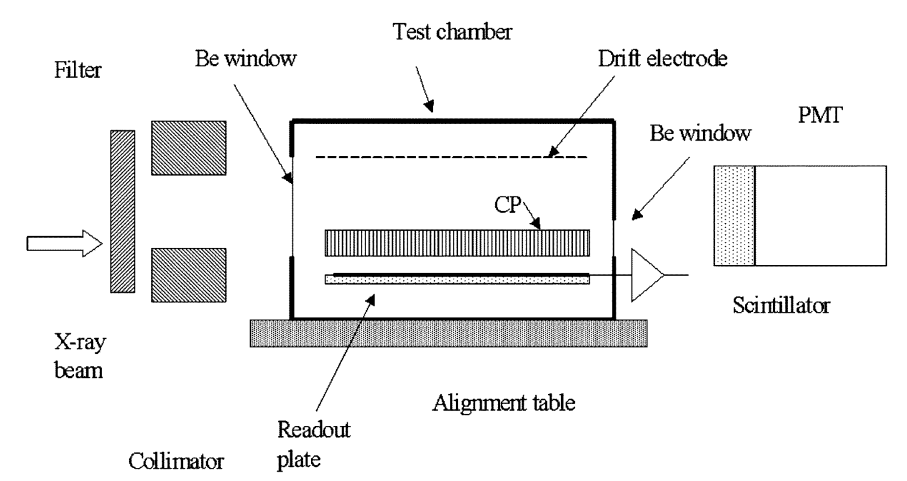

Fig. 2. Schematic drawing of the experimental set up for measurements of the efficiency of the hybrid gaseous detectors with respect to the PMT combined with a scintillator.

2) An edge-on illuminated CP may have a higher efficiency than the top illuminate one. Thus one can consider the application of the edge-on illuminated thin-wall CP's for the scanning systems.

Measurements described below were oriented on experimental verifications of these expectations.

\section{EXPERIMENTAL STUdY OF THIN-WALL CPS AS X-RAY CONVERTERS}

\section{A. Experimental Set Up}

Our experimental set up is shown schematically in Fig. 2. Essentially it contains a test chamber and an X-ray gun with a tungsten anode. In most measurements a filter and a slit collimator were placed in front of the chamber. The filter was used not only as an adjustable X-ray attenuator, but also (in the case of the strong absorption in it) allowed one to obtain a quasi monochromatic X-ray beam (see below). If necessary, a linear or a mammographic phantom (or any other object) could be installed in between the collimator and the test chamber. The test chamber was installed on a special step motor controlled table which allowed for 3D alignment to take place with up to a few $\mu \mathrm{m}$ in accuracy. The table also allowed chamber rotation for up to $90^{\circ}$.

Inside the test chamber various CP's and gas multiplication structures could be installed (Fig. 2). The CP's tested were made of lead glass and had thicknesses of 0.8-1 mm; the holes had diameters of 12,30 , and $100 \mu \mathrm{m}$ and the wall thickness was of 2.5, 6 , and $20 \mu \mathrm{m}$, respectively. Two to three $\mathrm{cm}$ above the $\mathrm{CP}$ a mesh was installed. If necessary, the gap between the mesh and the CP could be used as a drift region for the primary electrons created in that region by the X-ray radiation. In some measurements a readout plate was also placed $0.4 \mathrm{~mm}$ below the $\mathrm{CP}$; it was a ceramic plate with $\mathrm{Cr}$ strips of a $50 \mu \mathrm{m}$ pitch. A voltage of $1-2$ $\mathrm{kV}$ could be applied between the $\mathrm{CP}$ and the readout plate. This made it possible for avalanche multiplication to be achieved in this region. The combination of the solid X-ray converters (the CP's) with the gas amplification structure was given the name of a "hybrid detector".

Depending on the measurements one of the two readout plates was used. The first one was used for the CP's efficiency measurements; it had all of the strips interconnected together and to a single charge sensitive amplifier (Fig. 2) or to a Keithley 


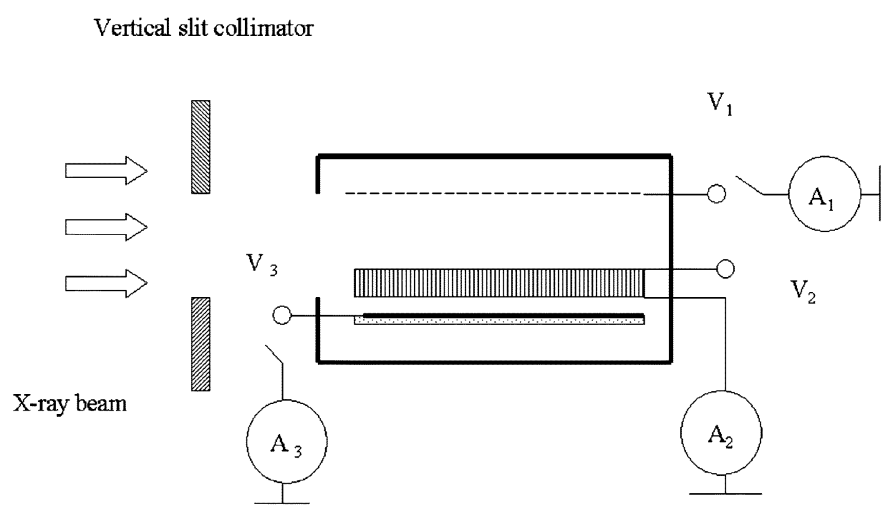

Fig. 3. Schematic drawing of the experimental set up oriented on measurements of currents from various electrodes and used also for measurements of the CP's efficiency in a current mode with respect to the efficiency of the gas.

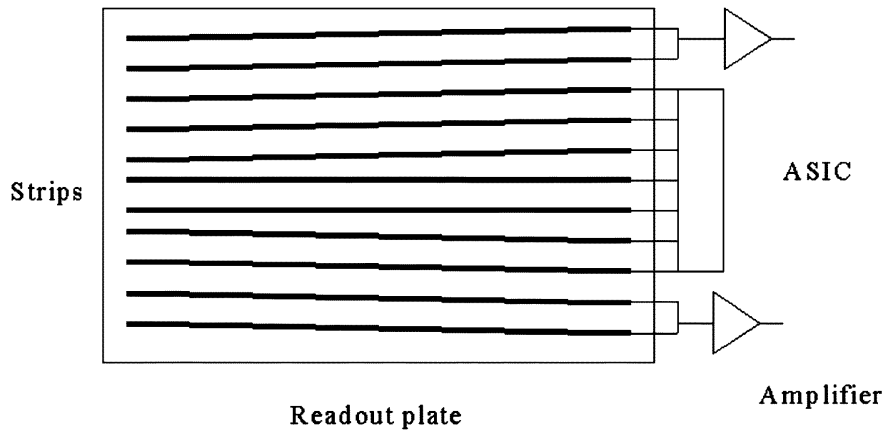

Fig. 4. Schematic drawing of the readout plate with the ASIC readout (top view). X-rays entered the $\mathrm{CP}$ (in edge-on illuminated mode) placed above the readout plate parallel to the strips. Primary electrons from the tracks created in the CP's holes after avalanche multiplication induced charge signals on these strips. Pulses from the strips were counted and the number of counts vs. the strip number were further used to form a 1D digital image of the transmitted X-rays through the object.

picoampermeter (Fig. 3). This allowed one to use this plate either for counting rate measurements (the counting rate $\mathrm{N}_{\mathrm{CP}}$ of the avalanches produced in the amplification region) or for various measurements of the current produced by the X-rays. The second readout plate was used for position resolution measurements; it had 120 strips in the central region of the plate connected to an ASIC-see Fig. 4. Other strips, either in groups or individually, were connected to charge sensitive amplifiers. In this plate, the strips were not parallel, but oriented at some angles to each other so that imaginary lines extended from these strips crossed at one point $78 \mathrm{~cm}$ apart from the readout plate ("focus point"). In position measurements the test chamber was aligned in such a way so that the focus point coincided with the $\mathrm{X}$-ray emitting sport from the X-ray gun. Signals from the ASIC passed the discriminators and were then simply counted one by one (digital readout); no any signal's interpolation methods (like the "centre of gravity") were applied. This not only simplified the electronics, but also allowed one to obtained images at a very high counting rate- up to $10^{5} \mathrm{~Hz}$ per strip. Charge sensitive amplifiers were used for analog measurements, for example to determine the induced charge profile on the anode strips or for measuring the pulse-height spectrum.

Behind the test chamber a photomultiplier tube (PMT) with a $\mathrm{NaI}$ scintillator was installed. When the chamber was removed

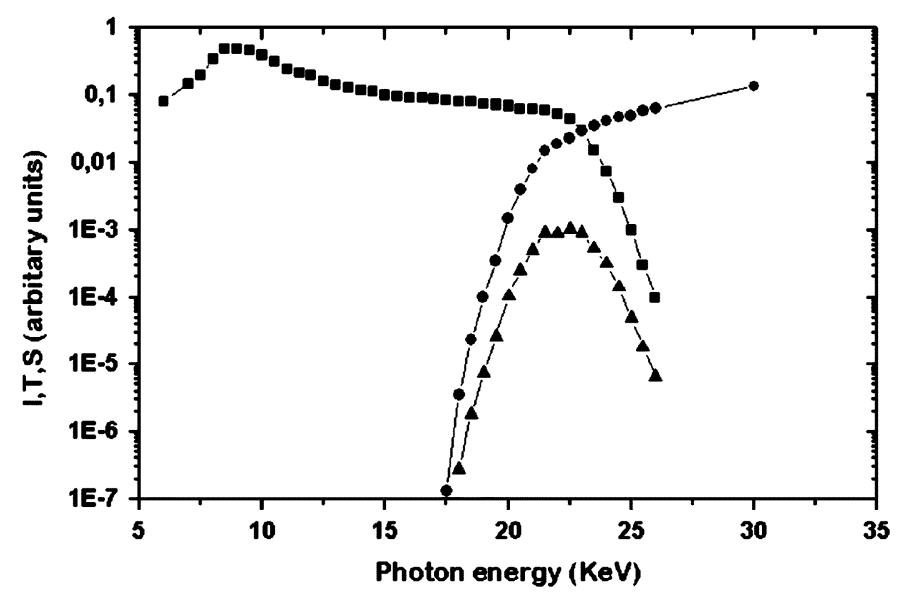

Fig. 5. Calculated emission spectrum I (photons/energy interval in arbitrary units) of the X-ray tube with a W anode at $\mathrm{V}_{\mathrm{g}}=26 \mathrm{keV}$ (triangles)[20], transmission $\mathrm{T}$ (arbitrary units) of the $\mathrm{Al} 0.75 \mathrm{~cm}$ thick (dots) [20] and the spectrum S (arbitrary units) of the X-rays passed the Al filter (squares). FWHM of this transmitted peak-type spectrum is $\sim 2 \mathrm{kV}$.

from the table, this detector was used for the measurements of the X-ray intensity mainly in the range of photon energies of $10-60 \mathrm{keV}$. For low intensity beams we also used a CdZnTe (CZT) detector.

It was essential in these studies to perform measurements of the CP's efficiency with a quasi monochromatic X-ray beam of a known energy: in the case of the unfiltered bremsstrahlung radiation at a given voltage $\mathrm{V}_{\mathrm{g}}$ applied to the $\mathrm{X}$-ray gun (15-60 $\mathrm{keV}$ ) the measured efficiency will be much higher due to the large contribution of photons with the energy $E_{\mathrm{v}}<V_{\mathrm{g}}$. To obtain the quasi monochromatic (narrow-band) X-ray beam the bremsstrachlung radiation from the gun was heavily filtered: in this case the radiation passing the filter had a rather narrow peak at photon energy $E_{\max }$ slightly below the voltage $V_{g}$ applied to the X-ray gun. This is because the transmission of a heavy adsorber is a very sharp function of the energy. This effect is illustrated by Fig. 5 .

One can see that independently of the exact shape of the emission's spectrum in the soft region (for simplicity some fine structure of the $\mathrm{W}$ emission spectrum was ignored), the transmission spectrum will have a sharp peak. The full width at half maximum (FWHM) of this peak-type distribution was, depending on the $\mathrm{V}_{\mathrm{g}}$ and the absorber thickness, $1-2.5 \mathrm{keV}$ - sufficient for our measurements. Thus after heavy filtering one can obtain an almost monochromatic beam with a characteristics energy $\mathrm{E}_{\max } \sim \mathrm{V}_{\mathrm{g}}$. The measurements with the CZT detector confirm these expectations for all voltages $\mathrm{V}_{\mathrm{g}}$ which could be applied to the X-ray gun (from 10 to $60 \mathrm{keV}$ ) [21].

\section{B. Measurements}

The CPs' efficiency for photons with energies of 30-60 keV was measured with respect to the PMT or CZT detectors. In this case the test chamber was placed $5 \mathrm{~m}$ away from the X-ray gun so that almost parallel beams of X-rays entered the detector's region. A vertical slit collimator $5 \times 30 \mathrm{~mm}^{2}$ was installed in front of the test chamber, aligned along the CP's diameter and along the axis of the PMT or the CZT detector. In this geometry only a fraction of the beam $1 / \mathrm{A}=\mathrm{L} / \mathrm{C}$ (where $\mathrm{C}$ is the distance 


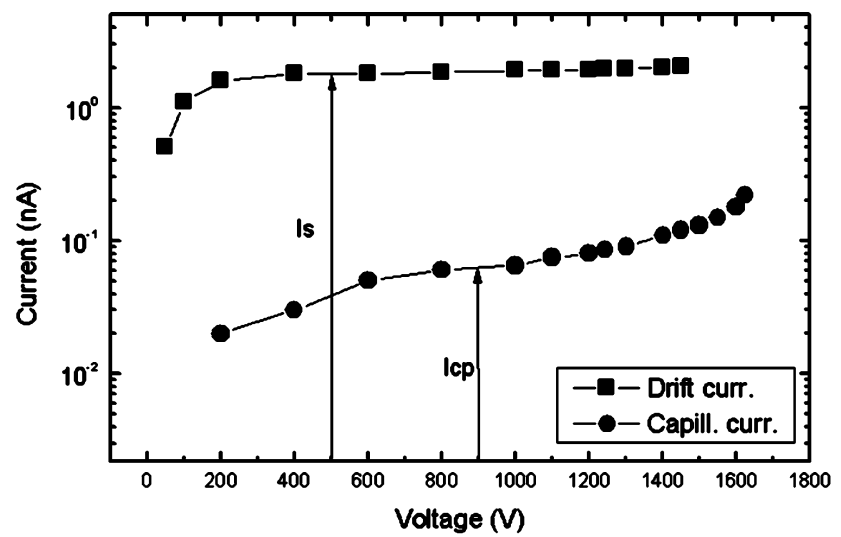

Fig. 6. Currents measured in the drift space (upper curve) due to the X-ray's interaction with the gas only and from the CP (lower curve) due to the X-ray's interactions with capillary walls. $\mathrm{V}_{\mathrm{g}}=15 \mathrm{keV}$. Gas mixture $\mathrm{Kr}+20 \% \mathrm{CO}_{2}$. Note that after correction on the geometrical factor (in our measurements $1 / \mathrm{A}=08 / 30$ ) the calculated efficiency of the $\mathrm{CP}$ will be 3 times higher than for the Kr-based gas mixture.

between the drift mesh and the $\mathrm{CP}$ ) hit the $\mathrm{CP}$ - see Fig. 2. The efficiency $\eta$ determined as

$$
\eta_{\text {edge }}=A N_{\mathrm{CP}} / N_{D}
$$

where $\mathrm{N}_{\mathrm{D}}$ is the counting rate from the PMT or the CZT for a heavily filtered $\mathrm{X}$-ray beam. The measurements were also done at various angles $\phi(\phi=0$ corresponds to the horizontal position of the $\mathrm{CP}$ ) between the X-ray beam and the CP. In this case

$$
\eta_{\phi}=\{C /(L \cos \phi+2 R \sin \phi)\} N_{\mathrm{CP}} / N_{D} .
$$

The cross checks of the efficiencies measured with the filtered bremsstrahlung radiation were also made with several X-ray radioactive sources: ${ }^{55} \mathrm{Fe}$ (main peak of the X-ray radiation at 5.9 $\mathrm{keV}$ ), ${ }^{109} \mathrm{Cd}$ (main peak of the $\mathrm{X}$-ray radiation at $22 \mathrm{keV}$ ) and ${ }^{241} \mathrm{Am}$ (main peak of the X-ray radiation at $60 \mathrm{keV}$ ). The Am source was always used in tandem with a filter suppressing soft $\mathrm{x}$-ray radiation from the source.

Current measurements mentioned above were performed in order to determine the CPs' efficiencies for soft $\mathrm{x}$-rays $(<30$ $\mathrm{keV}$ ) with respect to the know efficiency of the gas. They also allowed one to independently verify the measurements made with the PMT and the CZT detectors for the photons with the energy of $\leq 30 \mathrm{keV}$. This method of efficiency measurements is illustrated by Fig. 6 .

The upper curve represents the current [measured on the top electrode of the CP (Fig. 3)] vs. the voltage between the drift mesh and the CP's top when the detector was illuminated by an $\mathrm{X}$-ray beam. One can see that with the voltage the current first sharply increased and then reached the plateau (the saturated value of $\mathrm{I}_{\mathrm{S}}$ ). The lower curve shows the current measured on the CP's bottom electrode vs. the voltage across the CP. In these measurements the drift electrode was grounded. The behavior of this current is different from the previous one: first it increased, then reached the saturated value $\mathrm{I}_{\mathrm{CP}}$ (corresponding to full extraction of the electrons from the capillary holes-see [16] for more details) and then increased again (due to the avalanche gain in the $\mathrm{CP}$ ). These measurements allowed us to determine the ratio of the $\xi=\mathrm{I}_{\mathrm{s}} / \mathrm{I}_{\mathrm{CP}}$. The efficiency was determined as

$$
\eta=A\left\{1 / \xi\left(1-e^{-k x}\right)\right\}
$$

TABLE I

Some Results of the CPS EFFICIENCY MEASUREMENTS IN $\mathrm{He}+10 \% \mathrm{CH}_{4}$ Gas Mixture. Note That 22 and $60 \mathrm{keV}$ Photons Were Produced BY ${ }^{109}$ Cd AND ${ }^{241}$ Am SOURCES. PHOTONS OF OTHER ENERGIES Were From the Heavily Filtered X-Ray Gun Radiation. The VALUE OF $\phi=0$ CORRESPONDS TO THE X-RAY ENTERING THE CP

\begin{tabular}{|c|c|c|c|c|}
\hline $\begin{array}{l}\text { Capillary } \\
\text { type }\end{array}$ & $\begin{array}{l}\text { Efficiency } \eta(9) \\
\text { tube voltage }\end{array}$ & $\begin{array}{l}\text { for different } \\
\text { in } \mathrm{kV} \text { ): }\end{array}$ & gles $\varphi$ (data & racket-X-ray \\
\hline $\begin{array}{l}\text { Hole's } \\
\text { diameter, } \\
\mu \mathrm{m}:\end{array}$ & $\varphi=0^{\circ}$ & $\varphi=30^{\circ}$ & $\varphi=45^{\circ}$ & $\varphi=90^{\circ}$ \\
\hline 12 & $\begin{array}{ll}\eta=2.1 & (22) \\
\eta=12.8 & (35) \\
\eta=37 & (60)\end{array}$ & $\eta=9.3 \quad(30)$ & $\eta=7.5 \quad(30)$ & $\begin{array}{ll}\eta=3.2 & (10) \\
\eta=6.3 & (30) \\
\eta=3.75 & (60)\end{array}$ \\
\hline 30 & $\begin{array}{ll}\eta=5.2 & (35) \\
\eta=23 & (60)\end{array}$ & $\eta=3.8 \quad(35)$ & $\eta=3.2$ & $\begin{array}{ll}\eta=2 & (10) \\
\eta=2.8 & (35) \\
\eta \sim 1 & (60)\end{array}$ \\
\hline 100 & $\begin{array}{ll}\eta=0.1 & (10) \\
\eta=0.8 & (30) \\
\eta=6 & (60)\end{array}$ & $\eta=1.8 \quad(10)$ & $\eta=2.2 \quad(10)$ & $\begin{array}{ll}\eta=2.6 & (10) \\
\eta=1.5 & (30) \\
\eta=0.8 & (60)\end{array}$ \\
\hline
\end{tabular}
Parallel to Their Flat EleCtrodes, WhEREAS $\phi=90$ CORRESPONDS X-RAYS HitTING THE CPS FROM THE TOP

where $\mathrm{k}$ is a linear absorption coefficient in the gas for the $\mathrm{X}$-rays with the given energy $\mathrm{E}_{\mathrm{v}}$ and $\mathrm{x}$ is the thickness of the drift region. Such current measurements were done for each gas mixtures used: $\mathrm{Ar}+10 \% \mathrm{CH}_{4}, \mathrm{Xe}+10 \% \mathrm{CH}_{4}, \mathrm{Kr}+20 \% \mathrm{CO}_{2}$ or $\mathrm{He}+10 \% \mathrm{CH}_{4}$ at pressures of $1 \mathrm{~atm}$. The characteristic energy $\mathrm{E}_{\mathrm{v}}$ was determined from the convolution of the measured X-ray spectrum with the absorption curve of the gas. Typically $\mathrm{E}_{\mathrm{v}}$ was only slightly below $\mathrm{Vg}$.

\section{Results}

The operation of the converters was investigated in a wide range of X-ray energies (from 6 to $60 \mathrm{KeV}$ ) and at incident angles (from 0 to $90^{\circ}$ ). Some results of efficiency measurements in $\mathrm{He}+10 \% \mathrm{CH}_{4}$ mixture at $\mathrm{p}=1 \mathrm{~atm}$ are presented (Table I). This mixture was chosen because its stopping power was too low, so basically only CP's contributed to the measured efficiency. As one can see from this data the highest sensitivity was achieved with thin-wall capillaries (holes' diameter of 12-30 $\mu \mathrm{m}$ ) in an "edge on" illuminated mode.

Table II presents results of efficiency measurements for the edge on illuminated CP's in $\mathrm{Xe}+10 \% \mathrm{CH}_{4}$ gas mixture. One can see that compared to the He-based mixture, the efficiency for the soft X-rays increased due to the contribution from the gas itself.

We also tested multilayered $\mathrm{CP}$ converters illuminated from the top; in this case the efficiency could reach up to $50 \%$.

An induced charge profile on the anode strips and a position resolution (in digital form) were measured with a collimated $\mathrm{X}$-ray beam of $30 \mu \mathrm{m}$ in diameter entering the CP parallel to the readout strips. Some measured induced charge profiles are presented in Fig. 7(a); one can see that that their width slightly decreased with gain. In the case of the digital readout, the ASIC readout chip located for each event the strips nearer to the maximum of the induced charge profile (which was encompassed several strips) and counted the number of such hits for each strip. 
TABLE II

EFFICIENCY OF CPS MEASURED IN Xe $+10 \% \mathrm{CH}_{4}$ GAS MiXTURE AT $\mathrm{p}=1$ $\mathrm{atm}$ in EDGE ON ILLUMINATED GEOMETRY $(\phi=0)$ AND FOR X-RAYS HitTing THE CP FROM THE TOP $\left(\phi=90^{\circ}\right)$. IN THE CALCULATIONS OF $\eta$ CORRECTIONS WERE DONE ON THE ABSORPTION OF THE X-RAYS REACHING THE CP IN THE Xe+10\%CH4 Gas MiXTURE

\begin{tabular}{|c|c|c|c|}
\hline $\begin{array}{l}\text { Capillary } \\
\text { Type }\end{array}$ & \multicolumn{3}{|c|}{$\begin{array}{l}\text { Efficiency } \eta(\%) \text { for different angles } \varphi \text { (data } \\
\text { in bracket-X-ray tube voltage } V g \text { in } k V) \text {. }\end{array}$} \\
\hline $\begin{array}{l}\text { Hole's diameter in } \\
\mu \mathrm{m}\end{array}$ & $\varphi=0^{\circ}$ & $\varphi=90^{\circ}$ & \\
\hline 12 & $\begin{array}{l}\eta=23 \\
\eta=17.2 \\
\eta=43\end{array}$ & 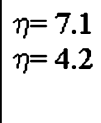 & $\begin{array}{l}(30) \\
(60)\end{array}$ \\
\hline 30 & $\begin{array}{ll}\eta=12.2 & (30) \\
\eta=26.1 & (60)\end{array}$ & $\begin{array}{l}\eta=3.2 \\
\eta=1.3\end{array}$ & $\begin{array}{l}(30) \\
(60)\end{array}$ \\
\hline 100 & $\begin{array}{ll}\eta=9.2 & \text { (30) } \\
\eta=11.8 & (60)\end{array}$ & $\begin{array}{l}\eta=2 \\
\eta=1.2\end{array}$ & $\begin{array}{l}(30) \\
(60)\end{array}$ \\
\hline
\end{tabular}

Usually the digital profiles were more narrow than the analogous one. As an example Figs. 7(b) and (c) show digital images of the X-ray beam in an enlarged scale allowing one an easier comparison with the induced charge profiles. One can see that at a gas gain of $\sim 10$ the position resolution of $150-250 \mu \mathrm{m}$ in digital form was achieved [Fig. 7(b)]. At gains of more than 1000 the position resolution improved to about $\sim 50 \mu$ [Fig. 7(c)]. This improvement was not only due to the more narrow induced charge profile, but also due to the fact that at high gains the detector operated in a noise free mode, when electronic noise pulses were fully discriminated. As was mentioned in the introduction, this is an important feature of the avalanche gaseous detectors operating in the photon counting mode [7]. Note also that result of these measurements were practically independent of X-ray energy.

For comparison we also performed the position resolution measurements for the X-ray beam entering the $\mathrm{CP}$ from the top. In this case both the induced charge profiles and digital images of the beam had long tails in the lower part of their profiles due to the contribution from the charge track created in the drift region. Thus, one can use the CP's in the X-ray imaging even in the case of the X-rays entering the CP's perpendicular to their flat surfaces, however a special care should be taken to minimize the thickness of the gas gap above the CP.

\section{X-RAy Detector BASED ON "EDGE ON" ILLUMINATED CPS}

Based on the results described above we have built a simplified prototype of an X-ray scanner which is shown schematically in Fig. 8. A lead glass CP $0.8 \mathrm{~mm}$ thick and with the holes diameter of $12 \mu \mathrm{m}$ was used in the "edge on" illuminated mode as an X-ray converter. A horizontal slit collimator $0.1 \times 7 \mathrm{~mm}^{2}$ was placed outside the test chamber-see Fig. 8.

The alignment of the slit with respect to the CP was performed by obtaining and analysing 1D digital images of the slit.
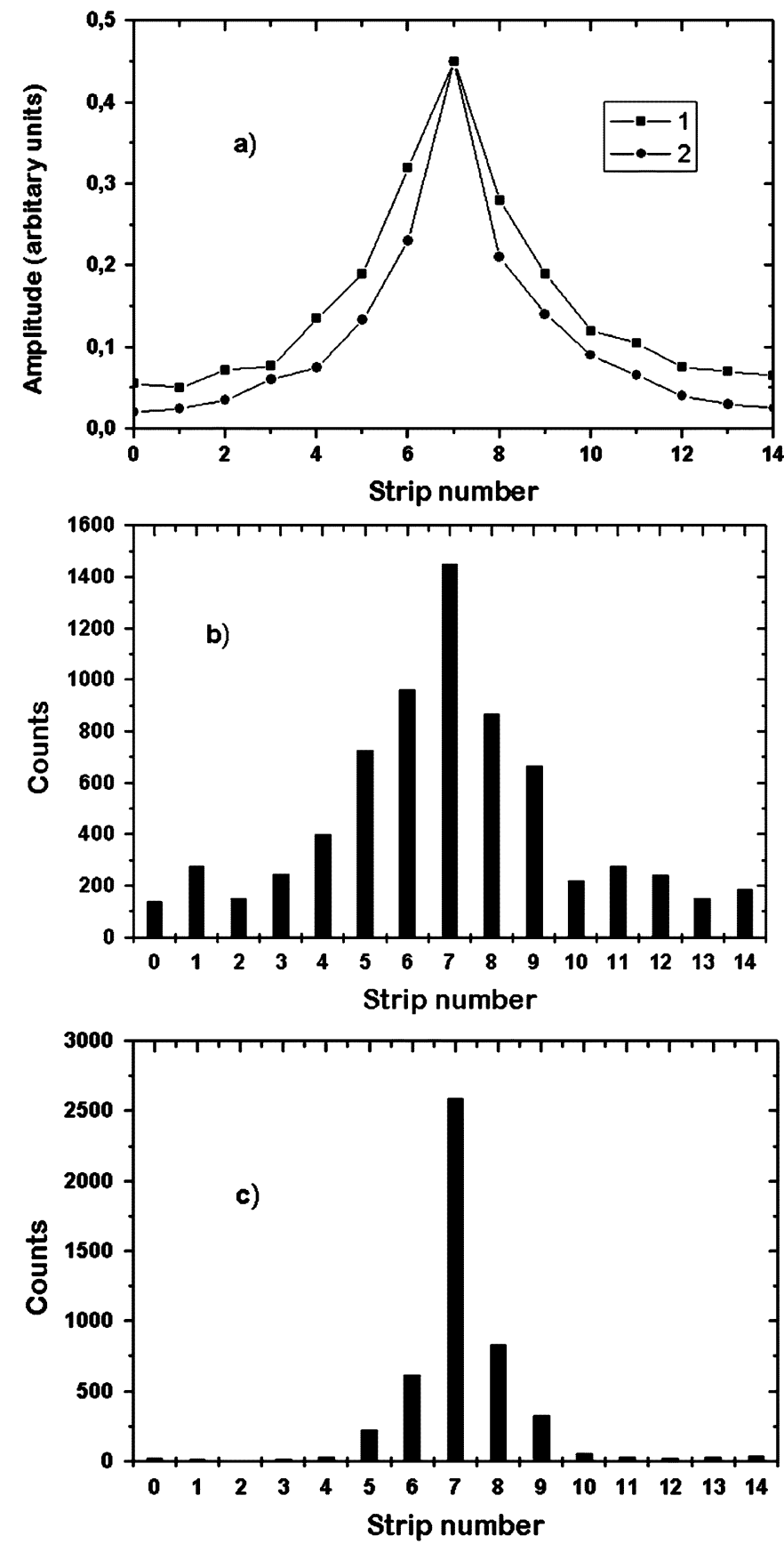

Fig. 7. Some results of the position resolution measurements made with a pencil beam $30 \mathrm{keV}$ X-rays: (a) induced charge profile measurements at gas gains $\sim 10$ (1) and $\sim 1000$ (2), (b) digital image of the X-ray beam at gain of 10 , (c) digital image of the $\mathrm{X}$-ray beam at gain of 10 . Gas mixture $\mathrm{Xe}+10 \% \mathrm{CH}_{4}$ at $\mathrm{p}=1 \mathrm{~atm}$.

The chamber was rotated and moved in two directions until the digital image of the slit becomes rectangular and the counting rate from each strip at the given voltage and the given current from the X-ray tube reached the maximum. After the alignment, a mammographic or a linear phantom was placed in between the slit collimator and the test chamber. One could then get series of 1D images of these phantoms at their various vertical positions. The measurements were performed at gas gains of about $3 \cdot 10^{4}$. As examples, Figs. 9-11 show some panoramic images: a mammographic phantom, a linear phantom and a slit $50 \mu \mathrm{m}$ 


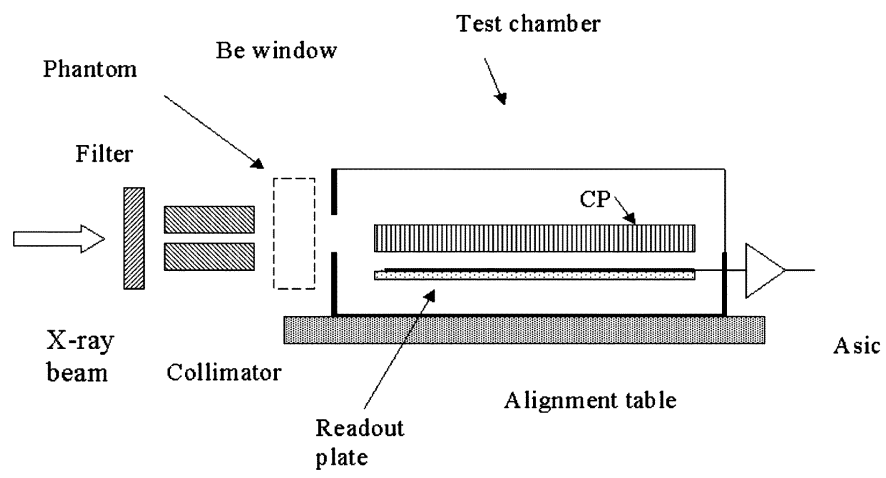

Fig. 8. Schematic drawing of the experimental set up for measurements of the position resolutions of the hybrid gaseous detectors.

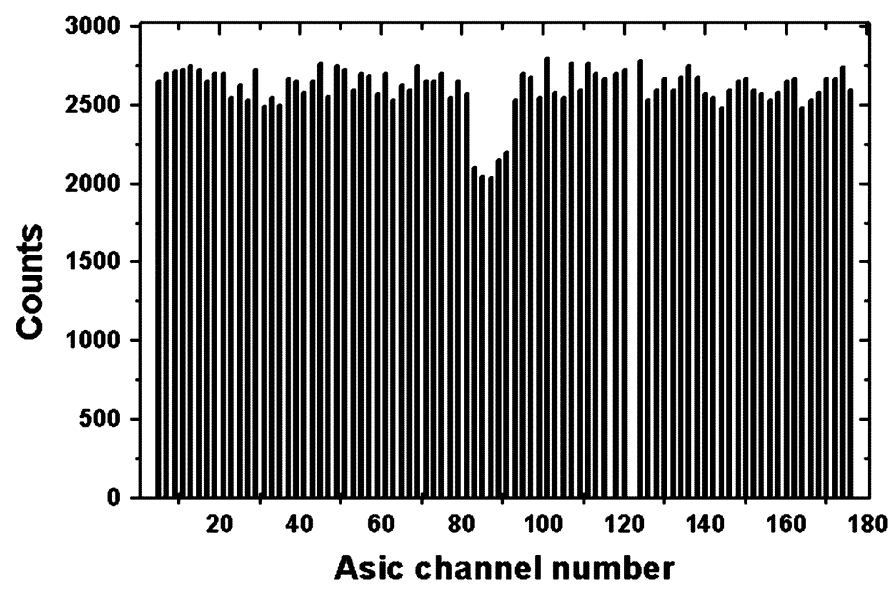

Fig. 9. On line digital image of a microcalcification $(0.54 \mathrm{~mm}$ size $)$ from a mammographic phantom. On the abscissa the strip's number is indicated, the ordinate shows the number of counts from these strips. Gas mixture $\mathrm{Xe}+10 \% \mathrm{CH}_{4}$ at $\mathrm{p}=1 \mathrm{~atm}$.

in width. In these figures the numbers on the $\mathrm{X}$-axis show the ASIC channel numbers and the numbers on the Y-axis show the measured counting rates.

As one can see from Figs. 10 and 11 position resolutions of $\sim 50 \mu \mathrm{m}$ were achieved in digital mode. This confirms the results of position resolution measurements performed with a 30 $\mu \mathrm{m}$ collimator and described above. The efficiency of the detector in these measurements was $\sim 20 \%$ in a good agreement with the results presented in the Table II.

\section{Discussion AND CONCLUSIONS}

The obtained images demonstrate the potentials of the new detector. One can see from these images that at gains of $3 \cdot 10^{4}$ the position resolution of $50 \mu \mathrm{m}$ was achieved in single photon counting (digital) mode. As follows from our earlier results, even better resolutions could be achieved with the readout plate having a strip's pitch of $30 \mu \mathrm{m}$ [15]. This position resolution is sufficient for some medical applications such as radiography or mammography.

The achieved efficiency $20-40 \%$ for $20-60 \mathrm{keV}$ X-rays could be further increased by using CP's with larger diameters or large-area rectangular CP's. Such hybrid detectors will combine

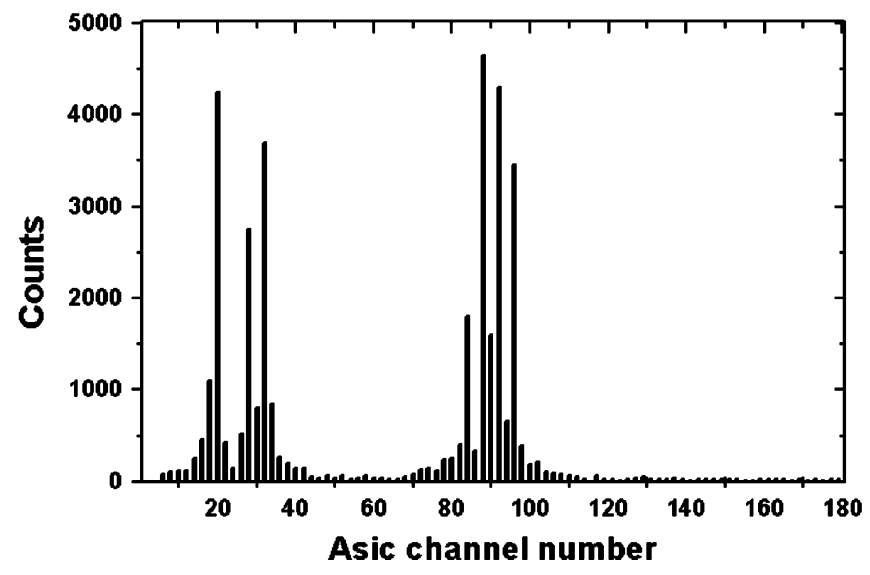

Fig. 10. Digital images of a linear phantom with 5.5 (left) and 6 (right) $1 \mathrm{p} / \mathrm{mm}$. On the abscissa the strip's number is plotted, Y-axis shows counting rate. Gas mixture $\mathrm{Xe}+10 \% \mathrm{CH}_{4}$ at $\mathrm{p}=1$ atm.

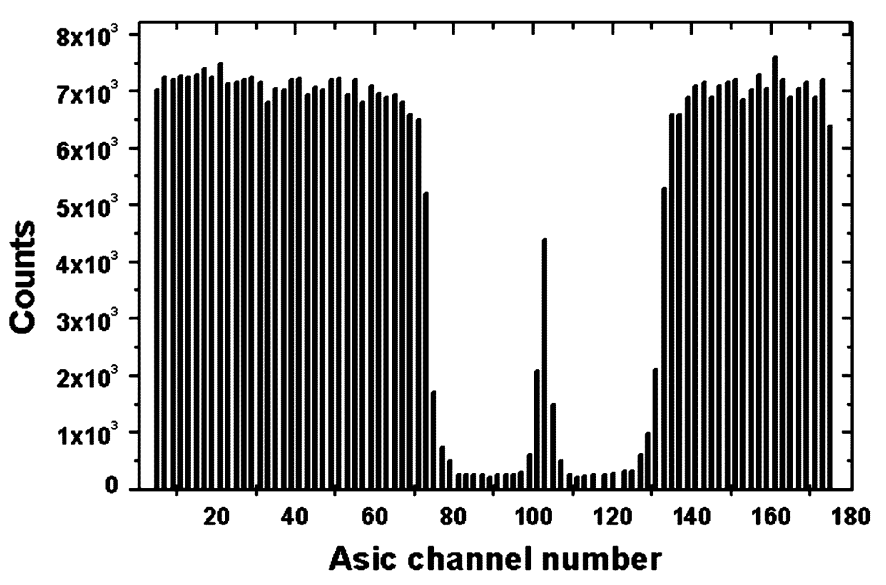

Fig. 11. On line digital image of two wires $50 \mu \mathrm{m}$ apart from each other. On the abscissa the strip's number is indicated, the ordinate shows the number of counts from these strips. Gas mixture $\mathrm{Xe}+10 \% \mathrm{CH}_{4}$ at $\mathrm{p}=1 \mathrm{~atm}$.

the advantages of high stopping power and a high position resolution typical for solid-state detectors with high avalanche multiplications offered by gaseous detectors.

Note that usual lead glass capillary plates can operate without noticeable charging up effects at counting rates of 50-100 Hz/mm $\mathrm{mm}^{2}$ and hydrogen -treated capillaries-up to $10^{5} \mathrm{~Hz} / \mathrm{mm}^{2}$ [22].

The developed detector may have several important advantages over the other X-ray imaging devices. For example, in contrast to the 2D medical imaging detectors (see for example [1], [10]) it operates in photon counting mode, much less sensitive to the scattered $\mathrm{x}$-ray photons (due to the strong X-ray collimation) and certainly should be much cheaper. We also expect that our hybrid detector will be cheaper than the scanning systems based on linear matrix of the scintillators [2] or solid-state detectors [1]. The low price in turn, may open possibilities in building large-scale scanning systems. Finally, compared to the scanners based on high pressure MWPC's or micropattern gaseous detectors, our hybrid detector has better position resolution, simpler and cheaper design (since it operates at $1 \mathrm{~atm}$ ) and simpler electronics (since it operates in a counting mode and there is no need for signal processing based on analog-interpolation methods). 
We believe that the suggested detectors after some improvements will open new possibilities for medical imaging, for example in mammography (scanning mode), radiography (including security devices) and crystallography. Thin-wall multi-layered CP's irradiated perpendicular to their surfaces could also be used as efficient X-ray converters for portal imaging devices [22].

\section{REFERENCES}

[1] M. G Yaffe and J. A. Rowlands, "X-Ray detectors for digital radiography," Phys. Med. Biol., vol. 42, pp. 1-39, 1997.

[2] M. J. Yaffe, "Digital mammography-detector considerations and new applications," Nucl. Instr. Meth., vol. A471, pp. 6-11, 2001.

[3] M. G. Bisogni et al., "Performances of different digital mammography imaging systems: Evaluation and comparison," Nucl. Instr. Meth., vol. A546, pp. 14-18, 2005.

[4] F. Dubecky et al., "Digital X-ray portable scanner based on monolithic semi-insulating GaAs detectors: General description and first "quantum" images," Nucl. Instr. Meth., vol. A546, pp. 118-124, 2005.

[5] F. Arfelli et al., "The digital mammography program at the SR light source in Trieste," IEEE Trans. Nucl. Sci., vol. 44, no. 6, pp. 2395-2399, Dec. 1997.

[6] M. Li, M. S. Dixit, and P. C. Johns, "Photon-counting digital radiography using high-pressure Xe filled detector," Nucl. Instr. Meth., vol. A471, pp. 215-221, 2001.

[7] T. Francke et al., "Dose reduction in medical X-ray imaging using noise fre photon counting," Nucl. Instr. Meth., vol. A471, pp. 85-87, 2001.

[8] E. A. Babichev et al., "Photon counting and integrating analog gaseous detectors for digital scanning radiology," Nucl. Instr. Meth., vol. A419, pp. 290-294, 1998.

[9] A. Barr et al., "A high-speed, pressurised multi-wire gamma camera for dynamic imaging in nuclear medicine," Nucl. Instr. Meth., vol. A477, pp. 499-504, 2002.
[10] R. M. Harrison et al., "Digital radiography-A review of detector design," Nucl. Instr. Meth., vol. A310, pp. 24-34, 1991.

[11] T. Franke and V. Peskov, "Micropattern gaseous detectors," in Proceedings of the 42nd Workshop on Innovative Detectors for Supercolliders, Preprint Physics/0 404 035, E. Nappi and J. Seguinot, Eds. Erice, NJ, Apr. 2004, pp. 158-180. 2004.

[12] M. V. R. Groshev et al., "Digital mammography with multielectrode ionization chamber," Nucl. Instr. Meth., vol. A454, pp. 130-135, 2000.

[13] G. Charpak, J. Dere, Y. Giomataris, and P. Rebourgeard, "Micromegas, a multipurpose gaseous detector," Nucl. Instr. Meth., vol. A478, pp. 26-36, 2002.

[14] V. Peskov et al., "The study and optimization of new micropattern gaseous detectors for high-rate applications," IEEE Trans. Nucl. Sci., vol. 48, no. 4, pp. 1070-1074, Aug. 2001.

[15] M. Danielsson et al., "Novel gaseous detectors for medical imaging," Nucl. Instr. Meth., vol. A518, pp. 406-410, Feb. 2004.

[16] C. Iacobaeus, T. Francke, M. Danielsson, J. Ostling, and V. Peskov, "Study of capillary-based gaseous detectors," IEEE Trans. Nucl. Sci., vol. 51, no. 3, pp. 952-959, Jun. 2004.

[17] A. Del Guerra, C. Lim, D. Ortendahl, and V. Perez-Mendez, "Medical positron imaging with dense drift space multiwire proportional chamber," IEEE Trans. Med. Imaging, vol. MI-1, no. 1, pp. 4-11, Aug. 1982.

[18] H. Sakurai, T. Tamura, S. Gunji, and M. Noma, "A new type of proportional counter using a capillary plate," Nucl. Instr. Meth., vol. A374, pp. 341-344, Feb. 1996.

[19] P. Carlson et al., "Beyond the RICH: Innovative photosensitive gaseous detectors for new fields of applications," Nucl. Instr. Meth., vol. A502, pp. 189-194, Apr. 2003.

[20] XCOM: Photon Cross Sections Database [Online]. Available: http://physics.nist.gov/PhysRefData/Xcom/Text/XCOM.html, http://www.protechn.ru/en/products.software.xenergo.php.

[21] L. Ericsson and V. Peskov, "Measurements of the X-Ray Gun Emission Spectra with a CZT Detector," XCounter Internal Rep., 1999.

[22] J. Ostling, A. Brahme, M. Danielsson, T. Francke, C. Iacobaeus, and V. Peskov, "Study of hole-type gas multiplication structures for portal imaging and other high count rate applications," IEEE Trans. Nucl. Sci., vol. 50, no. 4, pp. 809-819, Aug. 2003. 